\title{
Wright, Okasha and Chandler on transmission failure
}

\author{
ABSTRACT \\ Crispin Wright has given an explanation of how a first time warrant can fall \\ short of transmitting across a known entailment. Formal epistemologists have \\ struggled to turn Wright's informal explanation into cogent Bayesian \\ reasoning. In this paper, I analyse two Bayesian models of Wright's account \\ respectively proposed by Samir Okasha and Jake Chandler. I argue that both \\ formalizations are unsatisfactory for different reasons, and I lay down a third \\ Bayesian model that appears to me to capture the valid kernel of Wright's \\ explanation. After this, I consider a recent development in Wright's account of \\ transmission failure. Wright suggests that his condition sufficient for \\ transmission failure of first time warrant also suffices for transmission failure \\ of supplementary warrant. I propose an interpretation of Wright's suggestion \\ that shields it from objections. I then lay down a fourth Bayesian framework \\ that provides a simplified model of the unified explanation of transmission \\ failure envisaged by Wright.
}

KEYWORDS: warrant; transmission of warrant; Bayesianism; transmission failure; Wright; Okasha; Chandler

\section{Introduction}

Wright (1985) has given an interesting explanation of how warrant (or epistemic justification) can fall short of transmitting across known entailment. This explanation has been re-proposed in Wright (2002, 2003 and 2004) in connection with the discussion of hot topics of epistemology and philosophy of mind, such as the analysis of Moore's proof of the existence of a material world and the McKinsey paradox. Formal epistemologists have struggled to turn Wright's informal explanation of transmission failure into cogent Bayesian reasoning, but no satisfactory formalization has yet been achieved. Since Wright's informal explanation is intuitively plausible and, on the other hand, Bayesianism arguably provides the most successful formal account of inductive reasoning we have today, working out this formalization is somewhat compelling - if a suitable model were obtained, both Wright's explanation and Bayesianism would probably gain 
in plausibility. Furthermore, a successful model might illuminate features of the logical and epistemic architecture of Wright's explanation that informal analysis leaves undisclosed.

Wright (2002, 2003, and 2004) gives an explanation of how first time warrant (i.e. warrant for a proposition not yet warranted) can fail to transmit across known entailment. Wright (2007) suggests that the same explanation can be extended to clarify how supplementary warrant (i.e. warrant intended to strengthen one already possessed for a proposition) can fail to transmit across known entailment. In the first part of my paper, I focus on first time warrant. I provide an accurate formalization of Wright's explanation of transmission failure of first time warrant more exactly, I will propose a formal representation that appears to me to retain the essential logical features of Wright's explanation. In the second part, I focus on transmission failure of supplementary warrant. Wright claims that transmission failure of first time warrant and of supplementary warrant are elicited by the satisfaction of the same condition. In support of this claim, I will provide a simplified Bayesian model of the unified explanation of transmission failure that Wright appears to have in mind.

Section 2 scrutinizes Wright's informal account of failure of transmission of warrant focusing on first time warrant. Section 3 analyses and criticises the Bayesian formalizations of Wright's account put forward by Okasha (2004) and Chandler (2009); both formalizations are argued to be unsatisfactory. Section 4 lays down a third Bayesian framework that improves upon Chandler's and appears to capture the valid kernel of Wright's explanation. Section 5 scrutinizes Wright's thesis that transmission failure of first time warrant and transmission failure of supplementary warrant depend on the same sufficient condition. I argue that this thesis is problematic; I propose an interpretation of Wright's condition for transmission failure that appears to elude the difficulties. Section 6 lays down a fourth Bayesian framework that provides a simplified model of Wright's unified account of transmission failure. Section 7 draws the conclusions of the paper. 


\section{Wright on failure of transmission}

Transmissibility of warrant across entailment is important for our cognitive processes because it makes the advancement of knowledge (or of warranted belief) through deductive reasoning possible. According to Wright:

A warrant is transmissible [across a valid argument] when we may envisage a logically non-omniscient but otherwise perfectly rational subject coming to believe [its conclusion] for the first time in a way which depends on their recognizing both the validity of the [argument] in question and that they possess a warrant for its premises.

(2002, p. 332).

While discussing transmission of warrant, Wright appears to conceive of warrant as a form of propositional (rather than doxastic) justification. A subject has propositional justification for $P$ if and only if, roughly, $P$ is worthy of being rationally believed or accepted by her, though she may actually not believe or accept $P .{ }^{1}$ In the above quotation, Wright refers to what a perfectly rational subject would come to believe. Wright's assumption - which I find plausible - is that if a perfectly rational being would come believe $P$ in a given epistemic context, then $P$ is worthy of being rationally believed in that context. Note that Wright focuses on warrant sufficient to rationally believe or accept a proposition. Hence, when Wright says that a proposition $X$ is warranted, Wright means that $X$ is sufficiently justified to be rationally believable or acceptable. And when Wright says that evidence $Y$ provides a warrant for $X$, he means that $Y$ provides sufficient justification to believe or to accept $X$. This emerges with clarity in the above quotation and in the numerous examples of transmission and failure of transmission given in Wright (2002, 2003 and 2004). (I discuss some of them below).

\footnotetext{
${ }^{1}$ Wright notoriously endorses a disjunctive notion of warrant that comprehends both a notion of evidential justification and Wright's complex concept of entitlement, where to say that a rational subject $S$ is entitled to accept $P$ means, roughly, that it is epistemically appropriate for $S$ to accept (or trust) $P$ without any evidence for it (cf. Wright 2004).
} 
In general, Wright (2002, 2003 and 2004) would seem to conceive of the type of warrant that transmits from a proposition $X$ to a known consequence $Z$ as a first time warrant for $Z$. The process of transmission envisaged by Wright seems to be this: as a rational subject $S$ receives a first time warrant from evidence $Y$ for $X$ and deduces $Z$ from $X, S$ thereby receives a first time warrant from $Y$ for $Z$. Wright (2007) has broadened the class of the cases of warrant transmission to encompass also those concerning supplementary warrant. Since Okasha and Chandler do not focus on supplementary warrant (and to simplify my discussion), I prefer to leave the analysis of these cases to the last sections of this paper.

Wright is careful in distinguishing transmission of warrant across known entailment from closure of warrant under known entailment. Closure merely says that 'whenever there is warrant for the premises of a (known) valid argument, there is warrant for the conclusion too' (Wright 2002, p. 332). Transmission is more specific. It says that 'to acquire a warrant for the premises of a valid argument and to recognize its validity is thereby to acquire - perhaps for the first time - a warrant to accept the conclusion' (ibid.). Wright believes or tends to believe that warrant is closed under known entailment though it does not always transmit across entailment (cf. ibid.). I will follow Okasha (2004) and Chandler (2009) in assuming that evidence or evidential warrant is generally a proposition. ${ }^{2}$

We could summarize Wright (2002, 2003 and 2004)'s characterization of transmission of warrant in this way: consider any propositions $E, P$ and $Q$. The warrant supplied by $E$ for $P$ transmits to $Q$ just in case the following conditional is non-vacuously true:

(TRANS) If $E$ is a first time warrant for $P$ and $P$ knowingly entails $Q$, then $E$ is a first time warrant for $Q$.

Here is one of Wright's examples of warrant transmission; consider the following reasoning:

\footnotetext{
${ }^{2}$ For a defence of the propositional conception of evidence see Williamson (2000).
} 


\section{(Toadstool)}

E. Three hours ago, Jones inadvertently consumed a large risotto of Boletus Satana.

P. Jones has absorbed a lethal quantity of the toxins that toadstool contains.

Therefore:

Q. Jones will shortly die.

(2002, p. 332).

Here $\mathrm{E}$ is a first time warrant for believing $\mathrm{P}$, and $\mathrm{P}$ knowingly entails Q. It seems intuitive that if these two claims are true, E counts as a first time warrant for believing Q. Indeed, a rational subject who acquired the warrant from $\mathrm{E}$ for $\mathrm{P}$ and deduced $\mathrm{Q}$ would thereby acquire a warrant for Q. So the relevant instance of (TRANS) appears satisfied.

Wright has put forward a condition sufficient for failure of transmission of warrant. This condition has been adduced by Wright to substantiate the thesis that Moore's proof of the existence of a material world is not cogent (see mainly Wright 2002), and to analyse the structure of various sceptical arguments (see mainly Wright 2004). In this paper, I will attempt no direct evaluation of Wright's diagnosis of Moore's proof, ${ }^{3}$ nor will I investigate the plausibility of construals of sceptical arguments proposed by Wright. Rather, I will venture a formal analysis of Wright's general explanation of transmission failure.

According to Wright, 'transmission may fail in a case when there is warrant for the premises in the first place only because the conclusion is antecedently [i.e. prior and independently] warranted' (2002, p. 332). It is not completely clear to me what Wright means here with the expression 'only because'. It is tempting to interpret it as a mere 'only if'. Indeed, this reading appears licensed by many passages in Wright (2002, 2003 and 2004). For instance, Wright writes: 'So the ground provided by $E$ for believing $P$ is not transmissible across the entailment from $P$ to $Q$. [For] it is only if you already have grounds for $Q \ldots$ that $E$ gives you reason to believe $P^{\prime}$ (2002, p. 334. The second emphasis is mine). I will follow Okasha (2004) and

\footnotetext{
${ }^{3}$ Wright's analysis is controversial; see for instance Pryor (2004). Wright has formulated a variant of the condition for transmission failure that I analyse in this paper that applies to non-propositional evidence and that has been used by him to try to defuse McKinsey's Paradox. See for instance Wright (2000).
} 
Chandler (2009) in accepting this reading of 'only because', as it is prima facie plausible and greatly simplifies my analysis. In Section 5, I will suggest another interpretation (compatible with my criticism of Okasha's and Chandler's formalizations). I suspect that Wright (2002, 2003 and 2004) simply oscillates between these two interpretations.

This is one of Wright's examples of failure of transmission (cf. 2003, p. 59). Suppose Jessica and Jocelyn are known to be indistinguishable twins. Consider this reasoning:

(Twins)

E. That girl looks just like Jessica.

$P$. That girl is actually Jessica.

Therefore:

Q. That girl is not Jocelyn.

Here P knowingly entails Q. In what circumstances could E count as a first time warrant for believing P? Only if - presumably - it is prior and independently warranted that Q. In this case, the warrant from $\mathrm{E}$ for $\mathrm{P}$ cannot transmit to $\mathrm{Q}$ as a first time warrant for the latter. Consider a rational thinker who acquired a warrant for $\mathrm{P}$ from $\mathrm{E}$ because she had antecedent warrant for believing Q. This thinker could not acquire a first time warrant for Q via deducing $\mathrm{Q}$ from $\mathrm{P}$. For the thinker would already possess a warrant for Q. So the warrant supplied by E for P cannot transmit to Q.

We can summarize Wright's conception of transmission failure by appealing to the principle of warrant transmission (TRANS). Transmission failure is instantiated whenever (TRANS) is false. Wright seems to claim that (TRANS) is false if it is true that (a) $E$ is a first time warrant for $P$ which knowingly entails $Q$, and (b) $E$ is a first time warrant for $P$ only if $Q$ is antecedently warranted. The conjunction of (a) and (b) is logically equivalent to: $E$ is a first time warrant for $P$ which knowingly entails $Q$, and $Q$ is antecedently warranted. In short:

(FAIL) The antecedent of (TRANS) is true and $Q$ is antecedently warranted. 


\section{Okasha's and Chandler's formalizations of Wright's account}

Epistemologists have tried to translate Wright's explanation of failure of transmission into cogent Bayesian reasoning. Okasha (2004) has put forward a Bayesian model that has been found flawed or extremely puzzling by Chandler (2009). I believe that Okasha's model if flawed, but my reasons are different from Chandler's. The latter has also proposed a new formalization that I find unsatisfactory because incomplete in an important respect.

Let us first consider Okasha's model. Loosely speaking, a Bayesian theory is any theory of inductive reasoning based on the probability calculus, where probability is typically interpreted subjectively - i.e. as measuring the degrees of confidence of the rational subject $S$. Precisely, the prior probability of $X, \operatorname{Pr}(X)$, gives the degree of certainty or confidence of $S$ in $X$. The conditional probability of $X$ given $Y, \operatorname{Pr}(X \mid Y),{ }^{4}$ provides the degree of credence of $S$ in $X$ when $Y$ 's truth is given to $S$. $Y$ confirms $X$ if and only if $\operatorname{Pr}(X \mid Y)>\operatorname{Pr}(X)$, and $Y$ confirms $X$ conditional on $Z$ if and only if $\operatorname{Pr}(X \mid Y \& Z)>\operatorname{Pr}(X \mid Z)$. Okasha's framework appears to be based on at least two modelling assumptions: first, Wright's notion of $Y$ being a warrant for $X$ and the Bayesian notion of $Y$ confirming $X$ can be translated into one another; second, Wright's claim that $Y$ is a warrant for $X$ only if $Z$ is antecedently warranted and the claim that $Y$ confirms $X$ conditional on $Z$ can be translated into one another. Okasha is certainly aware that these two formal translations are imperfect (I return to this below), but he would seem to accept them as simplifying assumptions to make his model work in the first place.

Okasha substantially believes that if we translate (FAIL) into the conjunctive condition stating that (I) $\operatorname{Pr}(P \mid E \& Q)>\operatorname{Pr}(P \mid Q)$, (II) $\operatorname{Pr}(P \mid E) \leq \operatorname{Pr}(P)$ and (III) $P \mid-Q$ (cf. 2004, p. 141), we can formalize Wright's analysis of transmission failure as follows:

\footnotetext{
${ }^{4}$ In this paper, I assume that the conditional probabilities like $\operatorname{Pr}(X \mid Y)$ are always well defined in the sense that $\operatorname{Pr}(Y)>0$.
} 
(I) $\operatorname{Pr}(P \mid E \& Q)>\operatorname{Pr}(P \mid Q)$. (E confirms $P$ conditional on $Q)$.

(II) $\operatorname{Pr}(P \mid E) \leq \operatorname{Pr}(P)$. $\quad$ (E does not confirm $P$ otherwise).

(III) $P \mid-Q$. $\quad(P$ entails $Q)$.

Therefore:

(IV) $\operatorname{Pr}(Q \mid E) \leq \operatorname{Pr}(Q) . \quad(E$ does not confirm $Q)$.

(Cf. 2004, p. 142).

Okasha shows that (IV) logically follows from (I)-(III). Okasha believes that 'the valid kernel of Wright's informal reasoning [of why the warrant from $E$ fails to transmit from $P$ to its consequence $Q]$ is ... captured in the proof above' (2004, p. 145).

Chandler (2009) has raised two objections against Okasha's model. The first objection appears to me - so to speak - immanent to that model. It contends that, once the modelling assumptions are accepted, the resulting framework seems not to have an expected feature necessary to formalize Wright's notion of transmission failure. Chandler's second criticism appears to me - so to speak - external to Okasha's model. For it directly questions the acceptability the modelling assumptions.

Let us examine the first objection. Chandler notices that Okasha takes (TRANS) - or a very close principle - to characterize informally the notion of warrant transmission (cf. 2009, p. 3). Since Okasha translates ' $Y$ is a warrant for $X$ ' into ' $Y$ confirms $X$ ', Chandler infers that Okasha's model implicitly includes the following formalization of (TRANS):

(TRANS*) If $\operatorname{Pr}(P \mid E)>\operatorname{Pr}(P)$ and $P \mid Q$, then $\operatorname{Pr}(Q \mid E)>\operatorname{Pr}(Q)$.

This principle says that if $E$ confirms $P$ and $P$ entails $Q$, then $E$ confirms $Q$. Chandler then points out that (TRANS*) is not false but rather vacuously true when Okasha's formalization of (FAIL) - i.e. (I), (II) and (III) - is true. For (TRANS*)'s antecedent embeds the logical negation of (II). Chandler concludes that, given this, it is extremely puzzling to understand how Okasha's model could formalize Wright's explanation of failure of transmission. 
It seems to me that Okasha can answer this criticism. Okasha is presumably committed to (TRANS*). Note however that (TRANS*) is a principle of transmission of unconditional warrant because the relations of confirmation in it are all unconditional. On the other hand, the warrant from $E$ for $P$ which does not transmit to $Q$ is - according to Wright - a warrant conditional on antecedent warrant for $Q$. Thus Okasha's proof seems to presuppose, not (TRANS*), but some principle of transmission of conditional warrant. Since (TRANS*) is irrelevant for Okasha's proof, it is not odd or puzzling that (TRANS*) is vacuously true when (I)-(III) are true.

Whatever principle of transmission of conditional warrant Okasha might presuppose, there is a reason - unnoticed by Chandler - to doubt that Okasha's proof formalizes Wright's explanation of transmission failure. Okasha takes (IV) - i.e. $\operatorname{Pr}(Q \mid E) \leq \operatorname{Pr}(Q)$ - to be the Bayesian counterpart of Wright's claim that the warrant supplied by $E$ fails to transmit from $P$ to $Q$ (cf. 2004, p. 141). I think this interpretation of (IV) is incoherent with Okasha's own assumptions. As said, Wright believes that in case of transmission failure, the warrant supplied by $E$ for $P$ depends on antecedent warrant for $Q$. Thus, for Wright, the warrant for $P$ that fails to transmit to $Q$ is conditional warrant - it is one that depends on antecedent warrant for $Q$. Failure of transmission is thus instantiated, for Wright, because while $E$ supplies a warrant for $P$ that depends on antecedent warrant for $Q, E$ supplies no warrant for $P$ 's consequence $Q$ that depends on antecedent warrant for $Q$. Consequently, given Okasha's assumption that ' $Y$ is a warrant for $X$ depending on antecedent warrant for $Z$ ' and ' $Y$ confirms $X$ conditional on $Z$ ' translate into one another, the Bayesian construal of Wright's claim that the warrant supplied by $E$ fails to transmit from $P$ to $Q$ should be this: $\operatorname{Pr}(Q \mid E \& Q) \leq \operatorname{Pr}(Q \mid Q)$, i.e. $E$ does not confirm $Q$ conditional on $Q$. By contrast, (IV) says that $E$ does not confirm $Q$ unconditionally. Considering this, Okasha's proof may still account for some type of transmission failure but it does not seem to capture the 
type of transmission failure that Wright has in mind. Note that it is a priori true that $E$ does not confirm $Q$ conditional on $Q$. For $\operatorname{Pr}(Q \mid E \& Q)=\operatorname{Pr}(Q \mid Q)=1$. In Section 6, I will exploit this identity to provide Wright's explanation with an alternative formal model.

Let us turn to Chandler's second objection. Since Okasha's modelling assumptions make warrant coincide with confirmation, the claim that $E$ alone (i.e. without antecedent warrant for $Q$ ) is no warrant for $P$ translates into the claim that $E$ does not confirm $P$, which is what (II) asserts. (II) is indispensable to derive (IV) in Okasha's proof. Chandler (2009, p. 3) argues, however, that (II) is false in Dretske's Zebra case, which Wright (2003) counts as a genuine example of transmission failure. This is Dretske's famous case:

(Zebra)

E. The animal in the enclosure is a black and white-striped four-legged equine creature.

P. The animal in the enclosure is a zebra.

Therefore:

Q. The animal in the enclosure is not a cleverly painted mule.

Here P knowingly entails Q. According to Wright, E can count as a warrant for believing P only if it is antecedently warranted that Q. Consequently, the warrant from E for P does not transmit to Q. Chandler emphasizes that one's learning E will slightly increase one's confidence in $\mathrm{P}$, though not to the point to make one believe or accept $\mathrm{P}$, event if one does not take $\mathrm{Q}$ as given. I find this very plausible. Learning $\mathrm{E}$ will increase a bit one's confidence in both $\mathrm{P}$ and $\sim \mathrm{Q}$ (i.e. 'The animal in the enclosure is a cleverly painted mule') without sustaining one's belief in either proposition. Since $\operatorname{Pr}(\mathrm{P} \mid \mathrm{E})>\operatorname{Pr}(\mathrm{P})$, (II) is false.

What's the point of this criticism? Chandler writes that if (II) is false in Zebra 'and one wants to remain charitable to Wright, this might further indicate that Okasha's interpretation of Wright is off the mark' $(2009$, p. 3). Chandler is quite cautious. The reason is presumably this: though (II) is false in Zebra, this fact alone does not entail that Okasha's model is 'off the mark'. For 
any model is by definition a simplified, approximated or idealised representation of its original. Thus it is acceptable - and indeed expected - that there are mismatches between models and their originals. The point of Chandler's criticism seems to me to be this: since (II) fails in a case of transmission failure described by Wright, any model that did not include (II) would be - all other things being equal - more accurate than Okasha's model, and thus it would be in this sense preferable to the latter.

Chandler's criticism can be strengthened by broadening the set of sample cases in which (II) is false. Chandler does not note - or at least does not say - that (II) appears false in most, if not all, examples of transmission failure described in detail by Wright (2002 and 2007). Consider for instance this case:

\section{(Election)}

E. Jones has just placed an $\mathrm{X}$ on a ballot paper.

P. Jones has just voted.

Therefore:

Q. An election is taking place.

$$
\text { (2002, pp. 133-134). }
$$

Here P knowingly implies Q. Wright would seem to maintain the following: suppose it is known that Jones may place an $\mathrm{X}$ on a ballot paper even if there is no election. This may be the case, for instance, because it is known that Jones lives in a society where there are electoral drills (i.e. practice elections), which are as frequent as real elections. In this case, E can count as a warrant for believing P only if, presumably, Q is antecedently warranted. Hence, E cannot count as a first time warrant for Q. I find this plausible. Note however that, like in Zebra, it is intuitive that one's learning E will increase one's confidence in $\mathrm{P}$, though not to the point to make one believe $\mathrm{P}$, event if one does not take $\mathrm{Q}$ as given. Hence the Bayesian should conclude that $\operatorname{Pr}(\mathrm{P} \mid \mathrm{E})>\operatorname{Pr}(\mathrm{P})$, which falsifies (II). A similar argument can be made for Twins, considered before, the examples 
of transmission failure dubbed Soccer in Wright (2002, pp. 333-335) and Airport in Wright (2003, p. 5). In all these cases (II) appears false.

Let us now turn to Chandler (2009)'s formalization. While discussing Chandler's first objection, I explained why Okasha's model appears to me seriously flawed. Hence, I think Chandler is actually justified in looking for a new model. Chandler intends to remain as faithful as possible to Wright's notion of warrant. As I have said, Wright believes that a proposition is warranted just in case it is rationally believable or acceptable. This leads Chandler to accept the following modelling assumption:

$X$ is a warrant for $Y$ if and only if $X$ raises the probability of $Y$ and does so over a relevant probability threshold $t \in[0,1)$ sufficient for rational belief or acceptance, i.e. $\operatorname{Pr}(Y \mid X)>$ $\operatorname{Pr}(Y)$ and $\operatorname{Pr}(Y \mid X)>t$.

(Cf. 2009, p. 4).

Note that this new assumption allows for a quite accurate formal translation of Wright's notion of warrant which eludes the problems raised by Chandler's second criticism. On this principle, $E$ can certainly increase the probability of $P$ even if $E$ is not a warrant for $P$, provided that the probability of $P$ given $E$ remains lower than $t$.

Chandler translates Wright's (TRANS) into a conditional equivalent to this:

$$
\begin{aligned}
& \text { (TRANS1) If (1a) } \operatorname{Pr}(P \mid E)>\operatorname{Pr}(P),(1 \mathrm{~b}) \operatorname{Pr}(P \mid E)>t \text { and (2) } P \mid-Q \text {, then (3) } \operatorname{Pr}(Q \mid E)> \\
& \quad \operatorname{Pr}(Q) .{ }^{5}
\end{aligned}
$$

(Cf. 2009, p. 4).

This conditional states that if $E$ confirms $P$ such that $P$ 's probability given $E$ exceeds the threshold for rational acceptance $t$, and $P$ entails $Q$, then $E$ confirms $Q$. (Note that since (1b) and (2) together entail $\operatorname{Pr}(Q \mid E)>t, E$ confirms $Q$ such that $Q$ 's probability given $E$ exceeds $t)$.

Chandler assumes that the triples of propositions $E, P$, and $Q$ that satisfy both the antecedent and the consequent of (TRANS1) exhibit transmission success; conversely, those that satisfy the

\footnotetext{
${ }^{5}$ Chandler (2009, p. 7) also provides a more sophisticated version of (TRANS1) that does not seem to me to really improve the latter. For the sake of simplicity, I prefer to stick to (TRANS1).
} 
antecedent but not the consequent result in transmission failure. (TRANS1) vindicates Wright's example of warrant transmission Toadstool. Suppose again E = 'Three hours ago, Jones inadvertently consumed a large risotto of Boletus Satana', $\mathrm{P}=$ 'Jones has absorbed a lethal quantity of the toxins that toadstool contains', and Q = 'Jones will shortly die'. It is intuitively true that (1a) $\operatorname{Pr}(\mathrm{P} \mid \mathrm{E})>\operatorname{Pr}(\mathrm{P})$ and (1b) $\operatorname{Pr}(\mathrm{P} \mid \mathrm{E})>t$. Furthermore, (2) $\mathrm{P} \mid \mathrm{Q}$, and it appears also true that (3) $\operatorname{Pr}(\mathrm{Q} \mid \mathrm{E})>\operatorname{Pr}(\mathrm{Q})$. Thus (TRANS1) is non-vacuously true in Toadstool - the latter turns out to be a case of transmission, as expected. Yet (TRANS1) is false in many other cases because, when (2) is satisfied, there are probability distributions that verify (1a) and (1b) while falsifying (3) for any chosen $t \in[0,1)$. So (TRANS1) allows for transmission failure too.

Chandler proposes a formalization of Wright's condition (FAIL) that can be reduced to this:

(FAIL1) The antecedent of (TRANS1) is true and (1) $\operatorname{Pr}(Q)>t$. (Cf. 2009, p. 5). There is an important sense in which (FAIL1) appears to formalize Wright's original condition (FAIL) more accurately than Okasha's (I)-(III). Remember that (FAIL) states that (TRANS)'s antecedent is true and $Q$ is antecedently warranted. The conjunction (I)-(III) translates the claim that $Q$ is antecedently warranted into the intuitively stronger claim that $Q$ 's truth is given - this is so because (I) says that $E$ confirms $P$ given $Q$. On the other hand, (FAIL1) translates the claim that $Q$ is antecedently warranted into the proposition that $(1) \operatorname{Pr}(Q)>t$, i.e. $Q$ 's prior degree of probability suffices for rational belief or acceptance. This appears more faithful to the original proposition.

We saw above that Chandler's modelling assumptions appear in general more accurate than Okasha's. Furthermore, we have just seen that there is at least one sense in which (FAIL1) is more accurate than Okasha's (I)-(III). Despite this, many could be disappointed by Chandler's model. Chandler points out that his formalization actually captures a number of features of 
Wright's informal framework. For instance, it offers a plausible probabilistic characterization of (TRANS) such that (TRANS) admits of exceptions, as Wright requires. Furthermore, cases of transmission failure such as Zebra turn out to have the probabilistic structure to qualify as exception, as required by Wright. ${ }^{6}$ And so on (cf. 2009, p. 5). Yet Chandler emphasizes that while (FAIL) is a condition sufficient for transmission failure - and Wright is silent about whether (FAIL) is also necessary for it - (FAIL1) is only necessary for transmission failure but not sufficient. In other words, it can be shown that, necessarily, (FAIL1) is true if (TRANS1) is false, but there are probability distributions that make (FAIL1) and (TRANS1) true at the same time for any chosen $t \in[0,1)$ (ibid.).

Perhaps the fact that (FAIL1) does not reproduce an important logical feature of (FAIL) is not in itself a reason to conclude that Chandler's framework is flawed. For we should assume that Chandler intends his model only to have the consequences he has checked it for. Unlike Okasha, Chandler does not assert that his framework is meant to capture 'the valid kernel of Wright's informal reasoning'. One might say that Chandler's model works as a model of Wright's explanation simply to the extent it does. Nevertheless, it seems to me that if we aim at a Bayesian vindication of Wright's overall explanation of transmission failure - as I do in this paper - we should look for a more complete model than Chandler's; one in which the formalization of (FAIL) turns out to be a condition sufficient for transmission failure.

\section{Improving on Chandler's formalization}

Chandler seems not to have noted that a modest revision of (TRANS1) can turn the resulting version of (FAIL1) into a new condition the satisfaction of which suffices for transmission

\footnotetext{
${ }^{6}$ This is so because in Zebra it is intuitive that (3) $\operatorname{Pr}(Q \mid E)>\operatorname{Pr}(Q)$ is false.
} 
failure. Let us replace (1a) and (3) with, respectively, (1a') $\operatorname{Pr}(P) \leq t$ and (3') $\operatorname{Pr}(Q) \leq t$. We obtain:

(TRANS2) If (1a') $\operatorname{Pr}(P) \leq t,(1 \mathrm{~b}) \operatorname{Pr}(P \mid E)>t$ and (2) $P \longmapsto Q$, then (3') $\operatorname{Pr}(Q) \leq t$.

This conditional says that if $P$ 's prior probability does not exceed $t$, while the probability of $P$ given $E$ does, and $P$ entails $Q$, then $Q$ 's prior probability does not exceed $t$. Replacing (1a) and (3) with (1a') and (3') is not ad hoc but appear to further increase the accuracy of the model. For Wright (2002, 2003 and 2004) principally thinks of the warrant that transmits from $P$ to $Q$ as a first time warrant for $Q$. Chandler seems not to pay sufficient attention to this fact. The process of transmission envisaged by Wright is apparently this: as a rational subject $S$ gets a first time warrant from $E$ for $P$ and deduces $Q$ from $P, S$ gets a first time warrant from $E$ for $Q$. Suppose then that $t$ is the threshold for rational belief. It is intuitively plausible that $S$ can get a first time warrant from $E$ for $P$ just in case (1a') $\operatorname{Pr}(P) \leq t$ and (1b) $\operatorname{Pr}(P \mid E)>t$, and that $S$ can get a first time warrant from $E$ for $Q$ just in case (3') $\operatorname{Pr}(Q) \leq t$ and $\operatorname{Pr}(Q \mid E)>t .^{7}$ (Note again that (1b) and (2) together entail $\operatorname{Pr}(Q \mid E)>t)$.

Let us test (TRANS2) on Toadstool. In Wright's example, it is intuitively true that (1a') $\operatorname{Pr}(\mathrm{P})$ $\leq t$ while (1b) $\operatorname{Pr}(\mathrm{P} \mid \mathrm{E})>t$. Furthermore, (2) $\mathrm{P}$ entails $\mathrm{Q}$, and it appears true that (3') $\operatorname{Pr}(\mathrm{Q}) \leq t$. Since (TRANS2) is non-vacuously true in Toadstool, the latter turns out to be a case of transmission, as expected. The fact that Toadstool verifies both (TRANS1) and (TRANS2) is a mere consequence of the fact that (1a') and (1b), in (TRANS2), entail (1a) in (TRANS1), and that (3'), (1b) and (2), in (TRANS2), entail (3) in (TRANS1). Thus, the satisfaction of the more

\footnotetext{
${ }^{7}$ This entails that $\operatorname{Pr}(Q \mid E)>\operatorname{Pr}(Q)$. So $E$ is a first time warrant for $Q$ only if $E$ confirms $Q$. Note that there is a sense in which $E$ can be said to be a warrant for $Q$ even if $\operatorname{Pr}(Q \mid E) \leq \operatorname{Pr}(Q)$ - this may happen, intuitively, when both $\operatorname{Pr}(Q)>t$ and $\operatorname{Pr}(Q \mid E)>t$ (see for instance Achinstein 2001). In these cases, I would say that $E$ counts as a new warrant for $Q-$ since $\operatorname{Pr}(Q \mid E)>t$ - but not as a first time warrant for $Q-\operatorname{since} \operatorname{Pr}(Q)>t$.
} 
accurate formalization (TRANS2) entails the satisfaction of the less accurate formalization

(TRANS1), while the reverse is false.

As we replace (TRANS1) with (TRANS2), we should also replace (FAIL1) with the following new condition:

(FAIL2) The antecedent of (TRANS2) is true and (1) $\operatorname{Pr}(Q)>t$.

Note that (FAIL2) is just the logical condition of falsehood of (TRANS2). Therefore, (FAIL2) is a condition sufficient for transmission failure - this is what we were looking for. Interestingly, since (FAIL2) describes the sole logically possible case in which (TRANS2) is false, we have a clear-cut attestation that (FAIL2) is also a condition necessary for transmission failure. This is one of the benefits of the Bayesian formalization. Provided that this model is faithful to the original, (FAIL2)'s informal counterpart (FAIL) should turn out to be a condition necessary for transmission failure. Indeed, that the satisfaction of (FAIL) is necessary for transmission failure does not appear implausible upon reflection: if warrant is closed under known entailment, ${ }^{8}$ whenever $P$ is warranted, $P$ 's known consequence $Q$ must be warranted too. Thus, if a warrant for $P$ turns out not to be a first time warrant for $Q$, this can only happen - presumably - because $Q$ is antecedently warranted. $^{9}$

The Bayesian framework constituted by (TRANS2) and (FAIL2) appears to me to capture the valid kernel of Wright's informal account of transmission failure based on (TRANS) and (FAIL). In the next section, I will suggest that Wright's informal condition for transmission failure might be logically stronger than (FAIL). I will argue, however, that even if this is true, (TRANS2) and (FAIL2) still capture the essential logical features of Wright's explanation.

\footnotetext{
${ }^{8}$ The probabilistic counterpart of this principle might say that if $P$ entails $Q$, then $\operatorname{Pr}(Q) \geq \operatorname{Pr}(P)$.

${ }^{9}$ Note that I am relying on the assumption that (TRANS) provides an adequate analysis of Wright's notion of warrant transmission. If (TRANS) were replaced by a stronger principle - for example, one that added that the warrant from $E$ for $P$ transmits to $P$ 's consequence $Q$ only if $Q$ is warranted by $E$ in virtue of $P$ being warranted by $E$ - there might be new reasons why the warrant from $E$ for $P$ can fall short of transmitting to $Q$.
} 
This model provides a formal rationale for examples of transmission failure described by Wright. Consider again the case called Twins. It is known that Jessica and Jocelyn are indistinguishable twins. $\mathrm{E}=$ 'That girl looks just like Jessica'. $\mathrm{P}=$ 'That girl is actually Jessica'. $\mathrm{Q}=$ 'That girl is not Jocelyn'. (2) $\mathrm{P} \perp \mathrm{Q}$, and it is intuitive that in normal circumstances (1a') $\operatorname{Pr}(\mathrm{P}) \leq t$ and that $\left(1 \mathrm{~b}^{\prime}\right) \operatorname{Pr}(\mathrm{P} \mid \mathrm{E})>t$ only if $(1) \operatorname{Pr}(\mathrm{Q})>t$. Suppose now that the relevant instance of (FAIL2) is actually true - so that (1a') $\operatorname{Pr}(\mathrm{P}) \leq t$, (1b') $\operatorname{Pr}(\mathrm{P} \mid \mathrm{E})>t$, (2) $\mathrm{P} \mid-\mathrm{Q}$ and (1) $\operatorname{Pr}(\mathrm{Q})>$ $t$. In this case, the correlated instance of (TRANS2) is false. Thus, warrant fails to transmit, in accordance with our intuition. The same formal explanation successfully applies to Zebra, Election, Soccer and Airport.

\section{Wright on transmission failure of supplementary warrant}

The cases of transmission and failure of transmission considered hitherto have all been cases in which first time warrant respectively transmits or fails to do so. Wright (2007, p. 36) has broadened the class of the cases of warrant transmission to encompass also those concerning supplementary warrant - namely, those in which a rational subject would arrive at strengthening the warrant sufficient to believe a proposition that she already possesses.

Though Wright is not explicit on this, he would presumably make transmission of supplementary warrant depend on a principle roughly like this:

(Trans) If $Q$ is already warranted, $E$ is a warrant for $P$ and $P$ knowingly entails $Q$, then $E$ strengthens the warrant for $Q$.

Any triple of propositions $E, P$ and $Q$ instantiates transmission of supplementary warrant if the related instance of (Trans) proves non-vacuously true. Conversely, any such triple instantiates failure of transmission of supplementary warrant if the related instance of (Trans) is false. Note that the second condition in (Trans)'s antecedent requires $E$ to be a warrant for $P$. This means 
that $E$ is either a first time warrant for $P$ or a supplementary warrant for $P$. In either case, if transmission is instantiated, this warrant for $P$ transmits to $Q$ as a supplementary warrant for $Q$.

Cases in which (Trans) is intuitively non-vacuously true are those in which the antecedent of (Trans) is satisfied to the effect that the warrant for $P$ from $E$ is stronger than the warrant already available for $Q$. Consider this example: suppose it is known that only one ticket out of 5,000 of a fair lottery has been bought by a person born in 1970, and all other tickets have been bought by older or younger people. Consider this reasoning:

(Lottery)

E. The lottery winner's passport certifies she was born in 1980.

P. The lottery's winner was born in 1980.

Therefore:

Q. The lottery's winner was not born in 1970 .

Given Q's high chance, Q appears warranted from the beginning. On the other hand, E counts as a very strong warrant for $\mathrm{P}$ - intuitively, one even stronger than the initial warrant for $\mathrm{Q}$. $\mathrm{P}$ entails Q. In this case (Trans) appears non-vacuously true. Hence, this is a case of transmission of supplementary warrant. Imagine a rational subject $S$ who possessed the initial warrant for Q. It seems true that if $S$ acquired the new warrant from $\mathrm{E}$ for $\mathrm{P}$ and deduced $\mathrm{Q}, S$ would thereby boost her initial warrant for Q.

(Trans) possesses a straightforward Bayesian formalization. If $t$ is the threshold for rational acceptance, the claim that $E$ strengthens the warrant already available for $Q$ can be turned into the claim that $\operatorname{Pr}(Q)>t$ and $\operatorname{Pr}(Q \mid E)>\operatorname{Pr}(Q) .{ }^{10}$ Given this construal, the simplest way to formalize (Trans) is presumably the following:

$$
\begin{aligned}
& \text { (Trans1) If (1) } \operatorname{Pr}(Q)>t,(1 \mathrm{a}) \operatorname{Pr}(P \mid E)>\operatorname{Pr}(P),(1 \mathrm{~b}) \operatorname{Pr}(P \mid E)>t \text { and (2) } P \mid-Q \text {, then (3) } \\
& \quad \operatorname{Pr}(Q \mid E)>\operatorname{Pr}(Q) \text {. }
\end{aligned}
$$

\footnotetext{
${ }^{10}$ I interpret Wright's claim that $E$ is a supplementary warrant that $Q$ for $S$ as saying that $E$ raises $S$ 's original confidence in $Q$. An alternative interpretation of the same claim (which I cannot analyse here) says that $E$ enlarges the set of $S$ 's independent grounds for $Q$, where $E$ could do this without raising $S$ 's confidence in $Q$.
} 
(Trans1) says that if the prior probability of $Q$ exceeds $t, E$ confirms $P$ such that $P$ 's probability given $E$ exceeds $t$, and $P$ entails $Q$, then $E$ confirms $Q$ such that $Q$ 's probability given $E$ exceeds $Q$ 's prior probability. It is easy to show that if the antecedent of (Trans1) is satisfied to the effect that $\operatorname{Pr}(P \mid E)>\operatorname{Pr}(Q)$ - namely, in informal language, if the warrant for $P$ from $E$ is stronger than the warrant already available for $Q-$ the consequent of (Trans1) is also satisfied. ${ }^{11}$ This vindicates formally the intuitive cases of transmission of the same type as Lottery. (Trans1) proves false in many cases. For when (2) is satisfied, there are probability distributions that verify (1), (1a) and (1b) while falsifying (3) for any chosen $t \in[0,1)$. So (Trans1) allows for failure of transmission of supplementary warrant.

Wright (2007, p. 36) finds it intuitively plausible that failure of transmission of supplementary warrant is engendered by the satisfaction of the same condition sufficient for failure of transmission of first time warrant. Wright believes that when this condition is fulfilled, neither could one acquire first time warrant for $Q$ by acquiring a warrant from $E$ for $P$ and deducing $Q$ from $P$, 'nor is it intelligible how such reasoning could strengthen a warrant for $Q$ which [one] already possessed' (ibid.).

Unfortunately, the thesis that transmission failure of supplementary warrant is engendered by the satisfaction of the same condition that elicits transmission failure of first time warrant seems to have a very implausible consequence. So far I have interpreted Wright's condition for failure of transmission of first time warrant as making essentially two claims: (a) $E$ is a first time warrant for $P$ which knowingly entails $Q$, and (b) $E$ is a first time warrant for $P$ only if $Q$ is antecedently warranted. The conjunction of (a) and (b) is equivalent to (FAIL), i.e. $E$ is a first time warrant for $P$ which knowingly entails $Q$, and $Q$ is antecedently warranted. (FAIL) would

\footnotetext{
${ }^{11}$ Since (2) $P \mid-Q$, then $\operatorname{Pr}(Q \mid E) \geq \operatorname{Pr}(P \mid E)$. Suppose $\operatorname{Pr}(P \mid E)>\operatorname{Pr}(Q)$. By transitivity, (3) $\operatorname{Pr}(Q \mid E)>\operatorname{Pr}(Q)$.
} 
seem to be the condition that Wright refers to in many passages about failure of transmission of first time warrant. Suppose now that (FAIL) is also a condition sufficient for failure transmission of supplementary warrant. Note that whenever the antecedent of the principle for failure of supplementary warrant (Trans) is satisfied in a way that $E$ is a first time warrant for $P,{ }^{12}$ (FAIL) is true. ${ }^{13}$ The consequence is that if (FAIL) is a condition sufficient for failure of transmission of supplementary warrant, it does too much: it prevents supplementary warrant for $Q$ from transmitting every time it depends on first time warrant for $P$. Since supplementary warrant of this type does transmit in countless cases - Lottery is just one example - (FAIL) is not acceptable as a condition sufficient for transmission failure of supplementary warrant. This difficulty affects also (FAIL)'s Bayesian counterpart (FAIL2). After all, (FAIL2) is true whenever the antecedent of the formal principle of transmission of supplementary warrant (Trans1) is satisfied such that (1a') $\operatorname{Pr}(P) \leq t,(1 \mathrm{~b}) \operatorname{Pr}(P \mid E)>t$ (i.e. such that $E$ is a first time warrant for $P$ ).

How can we explain this puzzling result? Perhaps Wright is simply mistaken: transmission failure of first time warrant and transmission failure of supplementary warrant are not elicited by the satisfaction of the same condition. But drawing this conclusion at this point, without checking alternative possibilities, would be ill-advised. I do not think that our problem stems from (Trans), which appears straightforward to me. ${ }^{14}$ A possible explanation is that (FAIL) does not perfectly reflect the condition for transmission failure that Wright has in mind. The problem might be, more precisely, that Wright's claim that the first time warrant from $E$ for $P$ depends on

\footnotetext{
${ }^{12}$ That is to say, whenever it is true that $Q$ is already warranted, $E$ is a first time warrant for $P$ and $P$ knowingly entails $Q$.

${ }^{13}$ To be precise (FAIL) says that $Q$ is already and independently warranted, while the antecedent of (Trans) says only that $Q$ is already warranted. It would seem to me, however, that if the warrant for $Q$ is acquired before getting the warrant from $E$ for $P$, the warrant for $Q$ is also acquired independently of the latter.

${ }^{14}$ Note that if the claim that $Q$ is antecedently warranted were moved from the antecedent of (Trans) to its consequent, this would not solve our problem: if (FAIL) were the condition sufficient for transmission failure, no first time warrant for $P$ given $E$ could possibly transmit across the entailment as a supplementary warrant for $Q$.
} 
$Q$ being antecedently warranted is not fully captured by the conditional (b) embedded in (FAIL). This diagnosis would seem to be corroborated by a passage in Wright (2007) which says that when the warrant for $P$ from $E$ depends on $Q$ being antecedently warranted, the strength of the warrant supplied by $E$ for $P$ cannot exceed the strength of the antecedent warrant for $Q$ (p. 36). It is clear that (b) - which merely says, literally, that if $E$ is a first time warrant for $P$, then $Q$ is antecedently warranted - does not entail this consequence. To obtain this result, there must be some additional link between the warrant afforded by $E$ for $P$ and the necessary antecedent warrant for $Q$. The latter warrant should perhaps in some sense ground the former warrant. A mere material conditional like (b) cannot do this job.

If this diagnosis is correct, (FAIL) is not the condition sufficient for failure of transmission of supplementary warrant that Wright has in mind. (FAIL) should be replaced with a logically stronger proposition that would no longer be true every time the antecedent of (Trans) is satisfied such that $P$ is first time warranted by $E .^{15}$ This would dissolve our problems.

Note however that if this diagnosis is correct, (FAIL) cannot be Wright's condition for transmission failure of first time warrant either - at least the one referred to in Wright (2007). For transmission failure of both types of warrant is claimed by Wright (2007) to depend on the same sufficient condition. Note that if (FAIL) is not Wright's condition for transmission failure of first time warrant, (FAIL2) cannot be a completely faithful formalization of that condition. Quite the opposite, it is intuitive that to formalize that condition, (FAIL2) should also be replaced with a logically stronger proposition. It does not seem to me, however, that the correctness of this diagnosis would really undermine the accuracy of the model of Wright's account of transmission failure of first time warrant based on (TRANS2) and (FAIL2) presented in the former section.

\footnotetext{
${ }^{15}$ The replacement for (FAIL) should at least entail the new consequence that the strength of the required antecedent warrant for $Q$ does determine an upper bound for the strength of the warrant for $P$ given $E$.
} 
That model would still capture the essential logical features of Wright's explanation. The reason is that (FAIL2) describes the sole logically possible case in which (TRANS2) is false. Thus, any stronger proposition one might substitute for (FAIL2) would add essentially nothing to the explanation of (TRANS2)'s falsehood which simply states that (FAIL2) is true.

\section{Towards a unified model}

In the above section, I have argued that if Wright's thesis that transmission failure of first time warrant and of supplementary warrant depend on the same condition is correct, then this informal condition must be logically stronger than (FAIL). However, I have not attempted to evaluate whether Wright's thesis is correct. One way to determine the truth-value of this thesis would be investigating whether there is a suitable replacement for (FAIL) capable of explaining both types of transmission failure. Let me try a different route here, more in line with the formal approach of my paper. I will provide a new Bayesian framework that makes use of loosened modelling assumptions - i.e. assumptions less accurate than those underlying (TRANS2) and (FAIL2) - which approximates to the unified explanation of transmission failure that Wright has in mind. It seems to me that the existence of a simplified model of this type can confer at least some degree of plausibility to Wright's thesis. Furthermore, this model will pave the way for a more thorough investigation to be carried out elsewhere. ${ }^{16}$ The key idea of the new formalization is borrowed from Okasha's model scrutinized in Section 3. Precisely, the idea is that of interpreting Wright's claim that a warrant $w$ depends on $Q$ being antecedently warranted into the simplified claim that $w$ depends on $Q$ being part of background information, where background information is the conjunction of all propositions whose truth is considered to be given.

\footnotetext{
${ }^{16}$ See Moretti and Piazza (2010).
} 
To begin with, let us reformulate the formal principle of transmission of first time warrant (TRANS2) by making background information $B$ explicit. We obtain:

(TRANS3) If $\left(1 \mathrm{a}^{*}\right) \operatorname{Pr}(P \mid B) \leq t,\left(1 \mathrm{~b}^{*}\right) \operatorname{Pr}(P \mid E \& B)>t$ and (2) $P \mid-Q$, then (3*) $\operatorname{Pr}(Q \mid B) \leq t$.

(TRANS3) says that if $P$ 's probability given $B$ does not exceed $t$, while the probability of $P$ given both $E$ and $B$ does exceed $t$, and $P$ entails $Q$, then $Q$ 's probability given $B$ does not exceed $t$. (TRANS3) can account for transmission of first time warrant in Toadstool. Indeed, Toadstool seems to require substantive background information $B$ (which I have left implicit in my discussion in Section 2). For example, we can reasonably assume that E (i.e. 'Three hours ago, Jones inadvertently consumed a large risotto of Boletus Satana') counts as a first time warrant for P (i.e. 'Jones has absorbed a lethal quantity of the toxins that toadstool contains') if background $\mathrm{B}$ includes relevant mycological and medical information, where the claim that $\mathrm{E}$ is a first time warrant for $\mathrm{P}$ given $\mathrm{B}$ translates into the claim that $\operatorname{Pr}(\mathrm{P} \mid \mathrm{B}) \leq t$ and $\operatorname{Pr}(\mathrm{P} \mid \mathrm{E} \& \mathrm{~B})>t$.

Let us also reformulate the formal principle of transmission of supplementary warrant (Trans1) by making background information $B$ explicit. We obtain:

(Trans2) If (1*) $\operatorname{Pr}(Q \mid B)>t,\left(1 \mathrm{a}^{* *}\right) \operatorname{Pr}(P \mid E \& B)>\operatorname{Pr}(P \mid B),\left(1 \mathrm{~b}^{*}\right) \operatorname{Pr}(P \mid E \& B)>t$ and (2) $P \vdash Q$, then $\left(3^{* *}\right) \operatorname{Pr}(Q \mid E \& B)>\operatorname{Pr}(Q \mid B)$.

(Trans2) says that if $Q$ 's probability given $B$ exceeds $t, E$ confirms $P$ given $B$ such that the probability $P$ given both $E$ and $B$ exceeds $t$, and $P$ entails $Q$, then $E$ confirms $Q$ given $B$. (Trans2) can account for transmission of supplementary warrant in Lottery. Indeed, Lottery would seem to depend on substantive background information $B$. For example, it is natural to describe the fact that Q (i.e. 'The lottery's winner was not born in 1970') is already warranted in this way: this is so because background information B entails that only one ticket out of 5,000 of a fair lottery has 
been bought by a person born in 1970. The claim that Q is already warranted given background B intuitively translates into $\operatorname{Pr}(\mathrm{Q} \mid \mathrm{B})>t$.

Let us now turn to the condition for transmission failure. Wright's informal condition asserts that the first time warrant supplied by $E$ for $P$ does not transmit to $Q$ (in the form of first time or supplementary warrant for $Q$ ) if $E$ is a warrant for $P$ that depends on $P$ 's consequence $Q$ being antecedently warranted. Though at present I am unable to clarify how Wright exactly intends the expression 'depends' in this context, I suggest interpreting Wright's condition as asserting that the first time warrant supplied by $E$ for $P$ does not transmit to $Q$ if $E$ is a warrant for $P$ given background B, where B entails $P$ 's consequence $Q .{ }^{17}$ We can formalize this condition as follows:

(FAIL3) (1a*) $\operatorname{Pr}(P \mid B) \leq t,\left(1 \mathrm{~b}^{*}\right) \operatorname{Pr}(P \mid E \& B)>t$, (2) $P \mid-Q$, and (2a) $B \mid-Q$.

(FAIL3) says that $P$ 's probability given only $B$ does not exceed $t, P$ 's probability given both $E$ and $B$ does exceed $t$, and $P$ and $B$ individually entail $Q$. Note that it is not the case that (FAIL3) is true whenever the antecedent (Trans2) is satisfied such (1a*) $\operatorname{Pr}(P \mid B) \leq t$ and $\left(1 \mathrm{~b}^{*}\right) \operatorname{Pr}(P \mid E \&$ $B)>t$ (i.e. such that $E$ is a first time warrant for $P$ given $B$ ). Thus (FAIL3) does not raise the logical difficulty raised by (FAIL) and (FAIL2).

(FAIL3) and (TRANS3) account formally for failure of transmission of first time warrant because the truth of (FAIL3) makes (TRANS3) false. Suppose (FAIL3) is true. Since (1a*), $\left(1 b^{*}\right)$ and (2) are true, the antecedent of (TRANS3) is true. Since (2a) $B \mid-Q$, then $\operatorname{Pr}(Q \mid B)=1$. It is thus false that $\left(3^{*}\right) \operatorname{Pr}(Q \mid B) \leq t$. Hence the consequent of (TRANS3) is false. As the antecedent of (TRANS3) is true and its consequent is false, (TRANS3) is false. QED. In this case, a rational subject $S$ who acquired a first time warrant from $E$ for $P$ conditional on $B$ and

\footnotetext{
${ }^{17}$ Wright believes that when the condition sufficient for transmission failure is satisfied, the strength of the warrant supplied by $E$ for $P$ cannot exceed the strength of the antecedent warrant for $Q$. Note that this is certainly the case if the truth of $Q$ is given as background information.
} 
deduced $Q$ from $P$ could not acquire a first time warrant for $Q$ given $B$, as $S$ would already be conclusively warranted to believe $Q$ given $B$.

(FAIL3) and (Trans2) formally explain failure of transmission of supplementary warrant too, as the truth of (FAIL3) makes (Trans2) false. Suppose (FAIL3) is true. Since (2a) $B \downarrow Q$, then $\operatorname{Pr}(Q \mid B)=1>t$. So $\left(1^{*}\right)$ is true. Since $\left(1 \mathrm{a}^{*}\right) \operatorname{Pr}(P \mid B) \leq t$ and $\left(1 \mathrm{~b}^{*}\right) \operatorname{Pr}(P \mid E \& B)>t$, then $\operatorname{Pr}(P \mid E$ $\& B)>\operatorname{Pr}(P \mid B)$. So $\left(1 \mathrm{a}^{* *}\right)$ is true. Furthermore, (2) $P \mid-Q$. Since $\left(1^{*}\right),\left(1 \mathrm{a}^{* *}\right),\left(1 \mathrm{~b}^{*}\right)$ and $(2)$ are all true, the antecedent of (Trans2) is true. Moreover, since (2a) $B \mid-Q$, then $\operatorname{Pr}(Q \mid E \& B)=$ $\operatorname{Pr}(Q \mid B)=1$. It is thus false that $\left(3^{* *}\right) \operatorname{Pr}(Q \mid E \& B)>\operatorname{Pr}(Q \mid B)$. Hence, the consequent of (Trans2) is false. As the antecedent of (Trans2) is true and its consequent is false, (Trans2) is false. QED. In this case, a rational subject $S$ who acquired a warrant from $E$ for $P$ conditional on $B$ and deduced $Q$ from $P$ could not strengthen the warrant already possessed for $Q$ given $B$, for $S$ would already be conclusively warranted to believe $Q$ given $B$.

Let us apply this model to Wright's example of transmission failure Election. E = 'Jones has just placed an $\mathrm{X}$ on a ballot paper'. $\mathrm{P}=$ 'Jones has just voted'. $\mathrm{Q}=$ 'An election is taking place'. Let us assume, like in the original version, that Jones may often place an $\mathrm{X}$ on a ballot paper even if no election is taking place - let us assume this to be part of background information B. Given B, it seems correct to say that E is a warrant for P only if Q is antecedently warranted. When $\mathrm{Q}$ is antecedently warranted, the first time warrant for P from E cannot transmit to $\mathrm{Q}$ as a first time warrant for Q. Wright also finds it intuitive that when Q is antecedently warranted, the first time warrant for P from E cannot transmit to Q as a supplementary warrant for Q. If the simplifying assumption that ' $\mathrm{Q}$ is antecedently warranted' translates into ' $\mathrm{B}-\mathrm{Q}$ ' is accepted, the model based on (FAIL3), (TRANS3) and (Trans2) formally explains why Election instantiates both transmission failure of first time warrant and transmission failure of supplementary warrant. Suppose then that (2a) B - Q is true. It is intuitively plausible that 
$\left(1 \mathrm{a}^{*}\right) \operatorname{Pr}(\mathrm{P} \mid \mathrm{B}) \leq t$ and $\left(1 \mathrm{~b}^{*}\right) \operatorname{Pr}(\mathrm{P} \mid \mathrm{E} \& \mathrm{~B})>t$. Note also that (2) $\mathrm{P} \mid-\mathrm{Q}$. Since $\left(1 \mathrm{a}^{*}\right),\left(1 \mathrm{~b}^{*}\right),(2)$

and (2a) are true, (FAIL3) is true. Thus, (TRANS3) is false because $\operatorname{Pr}(\mathrm{Q} \mid \mathrm{B})=1>t$. Failure of first time warrant is instantiated. Since (FAIL3) is true, $($ Trans2) is also false, as $\operatorname{Pr}(\mathrm{Q} \mid \mathrm{E} \& \mathrm{~B})=$ $\operatorname{Pr}(\mathrm{Q} \mid \mathrm{B})=1$. Thus failure of transmission of supplementary warrant is also instantiated.

Despite these interesting results, let me stress once again that the framework constituted by (FAIL3), (TRANS3) and (Trans2) provides only a simplified model of the unified explanation of transmission failure that Wright seems to have in mind, and this is so quite independently of the precise formulation of Wright (2007)'s sufficient condition for transmission failure, which I have not attempted to determine in this paper. The fact is that Wright believes that warrant fails to transmit if (among other things) $Q$ is antecedently warranted, and not if $Q$ is just antecedently given. This is the main reason I am not completely satisfied with my unified model of transmission failure.

\section{Conclusions}

Wright has put forward an explanation of how warrant can fall short of transmitting across entailment that may bear on various philosophical debates. In this paper, I have first focused on Wright's account of transmission failure of first time warrant. I have argued that Okasha's and Chandler's formalizations of this account are unsatisfactory for different reasons: while Okasha's model does not have the right features for reproducing Wright's explanation, Chandler's model is incomplete. I have proposed an alternative model that appears to capture the essential logical features of Wright's explanation. This model arguably boosts the credibility of both Wright's explanation and Bayesian methodology as an accurate tool of inductive reasoning. An interesting consequence of this model is that the condition sufficient for transmission failure is also a necessary condition for transmission failure. 
In the second part of the paper, I have focused on Wright's recent suggestion that transmission failure of first time warrant and transmission failure of supplementary warrant share a sufficient condition. I have put forward a probabilistic model that comes close to vindicating this unified explanation. While this model encourages or might encourage the belief that Wright's suggestion is a correct, it also calls for a more thorough future investigation of this matter.

\section{Acknowledgments}

I am very grateful to Ben Blumson, Dylan Dodd, Rachael Briggs, Berit Brogaard, David Chalmers, Franz Dietrich, Paul Dimmock, Filippo Ferrari, Patrick Greenough, Alan Hájek, Leon Leontyev, Aidan McGlynn, Robert Nola, Glen Pettigrove, Tommaso Piazza, Dennis Robinson, Joe Salerno, Wolfgang Schwartz, Katie Steele, Crispin Wright and Elia Zardini for illuminating discussions and substantive comments upon drafts of this paper. I am also very grateful to two anonymous referees of this Journal, audience at the AAP 2008 conference in Melbourne, the ANU, the University of Auckland, and the NIP of Aberdeen University.

\section{References}

Achinstein, P. (2001). The Book of Evidence. (Oxford: Oxford University Press).

Chandler, J. (2009). The transmission of support: a Bayesian re-analysis. Synthese (in press). DOI 10.1007/s11229-009-9570-4.

Moretti, L. and Piazza, T. When warrant transmits and when it doesn't. (Manuscript).

Okasha, S. (2004). Wright on the Transmission of Support: a Bayesian analysis. Analysis, 64, pp. 139-146. 
Pryor, J. (2004). What's wrong with Moore's argument? Philosophical Issue, 14, Epistemology, pp. 349-378.

Williamson, T. (2000). Knowledge and its Limits. Oxford: OUP.

Wright, C. (1985). Facts and Certainty. Proceedings of the British Academy, 71, pp. 429-472.

Wright, C. (2000). Cogency and Question-Begging: Some Reflections on McKinsey's Paradox and Putnam's Proof. Philosophical Issues,10, 140-163.

Wright, C. (2002). (Anti-)Sceptics Simple and Subtle: G. E. Moore and John McDowell. Philosophy and Phenomenological Research, 65, pp. 330-348.

Wright, C. (2003). Some Reflections on the Acquisition of Warrant by Inference. In S. Nuccetelli (Ed.), New Essays on Semantic Externalism and Self-Knowledge, pp. 57-77. Cambridge, Mass.: MIT Press.

Wright, C. (2004). Warrant for Nothing (and Foundations for Free?). Proceedings of the Aristotelian Society, Supplementary Volume, 78, pp. 167-212.

Wright, C. (2007). Perils of Dogmatism. In S. Nuccetelli (Ed.), Themes from G. E. Moore: New Essays in Epistemology and Ethics, pp. 25-48. Oxford: OUP. 